\title{
Investigations of the flow field inside a square cyclone separator using DPIV and CFD
}

\author{
Marek Wasilewski ${ }^{1, *}$, and Lakhbir Singh Brar $^{2}$ \\ ${ }^{1}$ Opole University of Technology, Faculty of Production Engineering and Logistics, \\ ul. Proszkowska 76, 45-758 Opole, Poland, \\ ${ }^{2}$ Mechanical Engineering Department, Birla Institute of Technology, Mesra, Ranchi 835215, India
}

\begin{abstract}
This study used CFD and DPIV to investigate the flow field inside a square cyclone. For CFD, turbulent flow was modelled using 3D RANS equations (with an RSM complementary model). The results of the analysis indicate that the flow inside a square cyclone has two constituents: an outer, free flow and an inner, forced flow in the centre, as with cyclones with the traditional structure. Furthermore, the results indicate that the corners of a square cyclone generate additional vortexes that cause pressure drop to increase (but may, at the same time, potentially improve separation efficiency). The two methods provided highly consistent results. DPIV may be a very good method for validating results obtained with $\mathrm{CFD}$, including for the analysis of flow fields.
\end{abstract}

\section{Introduction}

A review of subject literature and practical examples shows that there are many structural variants of cyclone separators. The most common ones are the solutions proposed by Stairmand, Lapple and Swift [1]. Many studies have been conducted concerning these geometries [e.g. 2-9]. However, there are relatively few studies that address a type of cyclone with an unusual geometry, i.e. the square cyclone. Square cyclones are a response to the Circulating fluidized bed (CFB) boiler technology. Along with the development of large CFB boilers, the oversized body of the traditional cyclone became a significant drawback due to the thickness of the fireproof wall, as it necessitates a long start-up time for the boiler. The first square cyclone to be introduced into CFB boilers was the Pyroflow COMPACT, produced by the Ahlstrom Corporation in the 1990s [10]. The square cyclone had a lower separation efficiency than its round counterpart, but it was smaller, had a shorter start-up time, and was simpler and less expensive to manufacture. Later on, a new variant of the square cyclone was developed and applied in CFB boilers that implemented water cooling and a curved inlet, which helped to accelerate the particles and, consequently, improve separation efficiency [11-13]. Fatahian et al. [14] proposed an unusual solution to improve the efficiency of the square cyclone. They introduced a laminarizer inside the inlet and the vortex finder and found that it had a positive effect on separation efficiency.

*Corresponding author: m.wasilewski@po.opole.pl 
In the past, cyclone separators were selected for the purposes of particular installations usually through experimental research and empirical and semi-empirical models. Such research was expensive and time-consuming. With the development of computer technology and software, new structural designs of cyclone separators began to rely extensively on research involving the computational fluid dynamics (CFD), which today constitutes the most popular method in studies on the subject. Various numerical models are employed, including k- $\varepsilon$ [e.g. 15-16], Reynolds Stress Model (RSM) [e.g. 3-6, 17-21] or Large-Eddy Simulation (LES) [e.g. 7, 8, 22, 23], depending on the assumed goals and scope of the study. However, this type of research needs to be validated against other methods in order to be considered reliable. Validation is most often performed based on traditional experimental research, which is limited by the fact that it only analyses separation efficiency and pressure drop. It is impossible to conduct an extensive analysis of the field distributions of the fluid phase flow or the trajectory of the solid phase.

This issue can be addressed by using Digital Particle Image Velocimetry (DPIV). Particle Image Velocimetry (PIV) was first applied outside the laboratory wind tunnel in the 1980s. At the time, obtaining a high-quality image took between two and three days. Today, thanks to modern high-speed CCD and CMOS digital cameras and high-capacity computer units, the image is ready within seconds [24, 25]. This is one of the reasons why the PIV (currently DPIV) is growing in popularity. The second reason is the possibility to obtain temporary velocity fields, which in turn allows for a quantitative detection of the spatial structures in unstable flows. The third reason is the need for experimental data used to verify CFD codes. The growing popularity of DPIV translates into an increasing number of studies (from many disciplines) that apply this method [e.g. 26-33]. Research on cyclone separators has not yet taken full advantage of DPIV: subject literature includes few studies on cyclones that use this method [34-36].

The aim of this study was to investigate of the flow field inside a square cyclone separator using DPIV and CFD. The study involved a cyclone with a nontraditional structure, i.e. a square cyclone. The DPIV method was verified (in relation to CFD research) in terms of vectors maps and mean tangential and axial velocities within selected planes. In addition, the values of pressure drop obtained through experimental research were compared to those obtained through CFD research.

\section{Experimental and DPIV studies}

For the purpose of the research, optical track was constructed (Fig. 1). The main component was the square cyclone separator (Fig. 2). Because the researched object needed to be made from a transparent material in order to conduct DPIV measurements, the model was made from poly(methyl methacrylate) (PMMA). The DPIV setup consisted of an Nd:YAG laser as the source of light, a CCD camera (placed at a distance allowing for full use of the available image resolution) that registered flow phenomena and an impulse generator to synchronize the other two devices. A seeding device was installed in front of the cyclone inlet to introduce seeding particles, i.e. Di-Ethyl-Hexyl-Sebacic-Acid-Ester (DEHS), into the measurement space. The setup was controlled through the Dantec Dynamic Studio $2015 \mathrm{~b}$ software. The same setup was used to record and to process the images and analyze them digitally. Table 1 presents the technical parameters of the DPIV setup.

In addition, measurement equipment (flow meter, pressure sensors + differential pressure gauge) and a transportation system for the fluid phase (pipe, exhaust fan with frequency converter) were installed. A transportation system for the solid phase was also installed in order to model the real two-phase flow (in this study, the share of the solid phase amounted to $0.005 \mathrm{~kg} / 1 \mathrm{~m}^{3}$ ). Measurements were performed at four different inlet 
velocities: $v_{i}=7 \mathrm{~m} / \mathrm{s}(R e=233,808), v_{i}=9 \mathrm{~m} / \mathrm{s}(R e=300,610), v_{i}=10 \mathrm{~m} / \mathrm{s}(R e=334,011)$ and $v_{i}=12 \mathrm{~m} / \mathrm{s}(\operatorname{Re}=334,011)$.

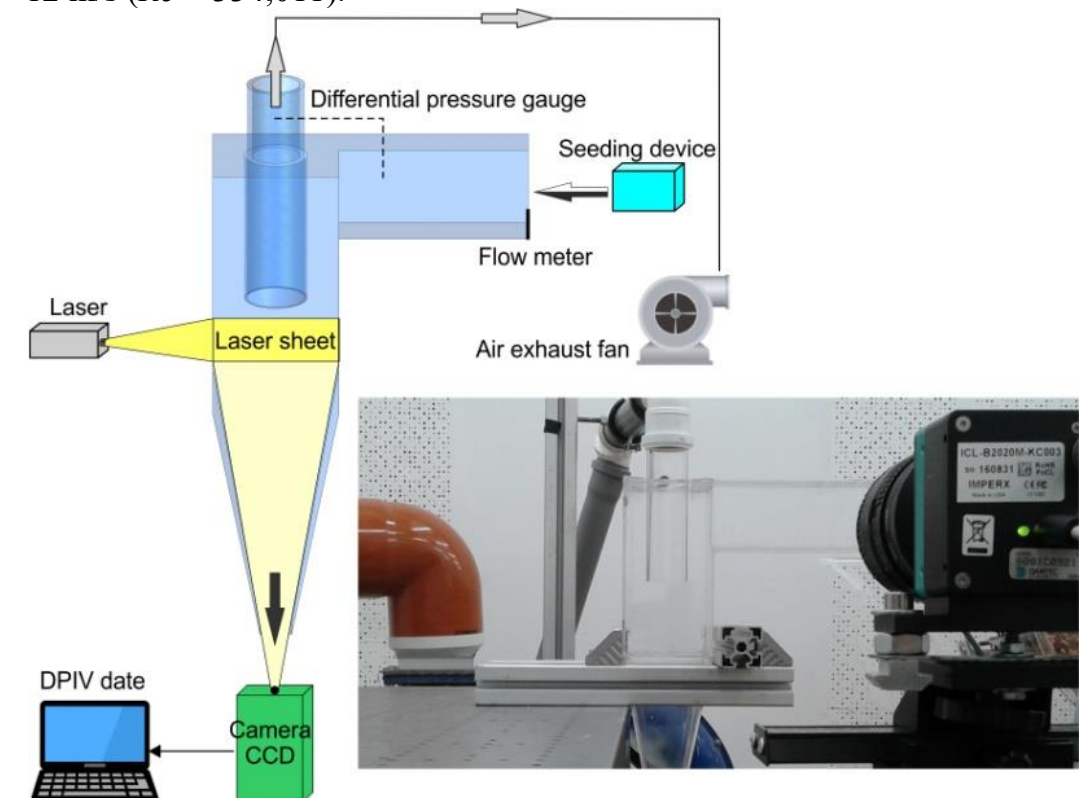

Fig. 1. Experimental set-up.

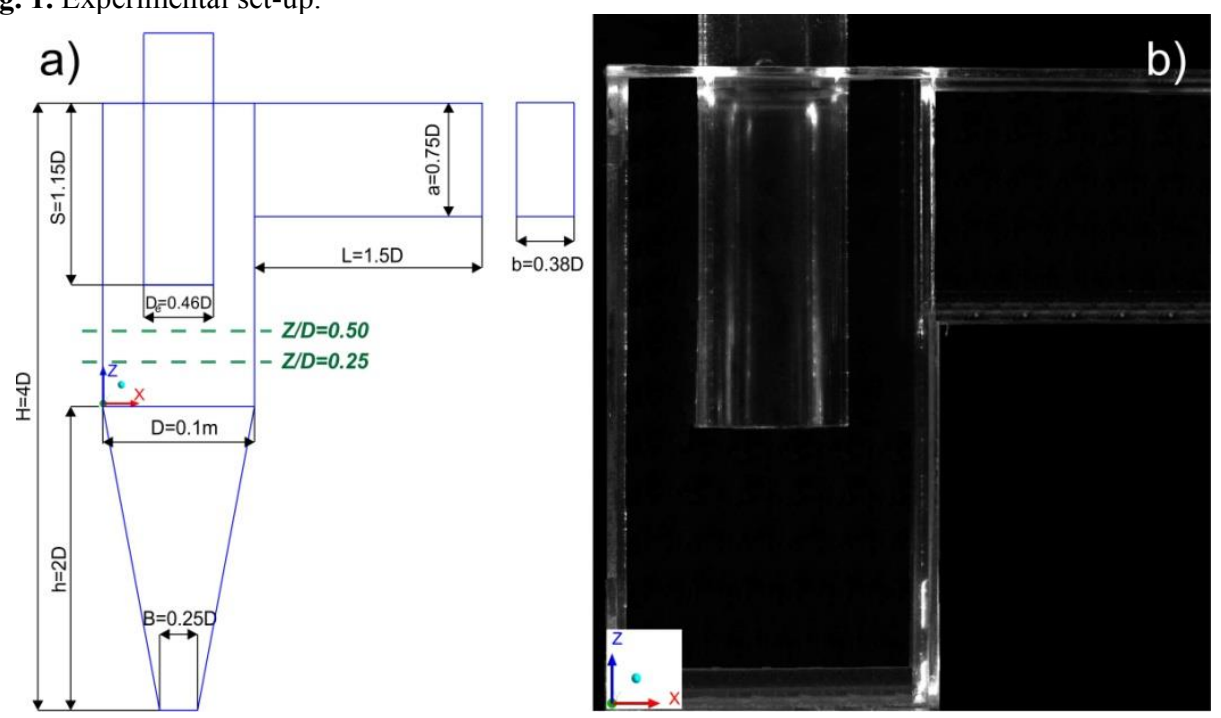

Fig. 2. Geometry of the cyclone separator (a) and view from the CCD camera (b).

Table 1. Technical parameters of DPIV system.

\begin{tabular}{|c|c|}
\hline Parameter & Description \\
\hline Camera CCD & Dantec Dynamics FlowSense EO 4M \\
\hline Laser & Dantec Dynamics DualPower TR \\
\hline Pulse generator & Berkeley Nucleonics Corp Model 575-8 \\
\hline Time between pulses, $\mu$ s & 25 \\
\hline Interrogation area size (horizontal x vertical), pix & 32 x 32 \\
\hline DPIV algorithm & $\begin{array}{c}\text { Adaptive Correlation with Local } \\
\text { Neighbourhood }\end{array}$ \\
\hline Detection method & Normalized median validation \\
\hline
\end{tabular}




\section{CFD studies}

The finite volume method based Fluent software (Version 18.2) was used to conduct the CFD research. The RANS method, coupled with the complementary RSM model, was used to model the turbulent flow. Detailed information about these models can be found in several studies as [3,18-21]. The solution was found by formulating equations for the pressure (Pressure Based Solution Method Segregated Solver). Differential equations were solved using the SIMPLE (Semi Implicit Method for Pressure Linked Equations) computational algorithm in order to correctly determine the coupling between the pressure and velocity fields and to maintain the momentum continuity equation. The second-order upwind interpolation method was used to determine the representative samples of the constituent values on the surface of the control volumes. The convergence condition was set at $10^{-6}$ for the continuity equation and $10^{-3}$ for the other equations. The flow conditions corresponded to the conditions applied in the experimental measurements. The 'velocityinlet' boundary condition was used for the inlet and the 'outflow' boundary condition was used for the outlet.

A model was created based on the geometry of the cyclone separator that was used in the experimental measurements. A three-dimensional domain was applied. In the next stage, the computational domain was discretized. A sensitivity analysis was conducted in order to take into account the effect of the density of the computational mesh. Three mesh densities were generated (Mesh 1 - about 240,000 elements, Mesh 2 - about 325,000 elements, Mesh 1 - about 392,000 elements). In each case, the computational area was discretized using a hexagonal mesh with a varying density depending on the area of the device. The value of the pressure drop was validated with respect to the experimental measurements. For Mesh 1, the difference between the measurement methods was about $11 \%$, regardless of the Re. For Meshes 2 and 3, the difference ranged from $8 \%$ to $9 \%$. Mesh 2 was selected for a further analysis due to its minimal density that did not display large changes in the obtained values. Figure 3 compares the obtained values of Euler number (Eu) for Mesh 2 depending on the Reynolds number $(R e)$.

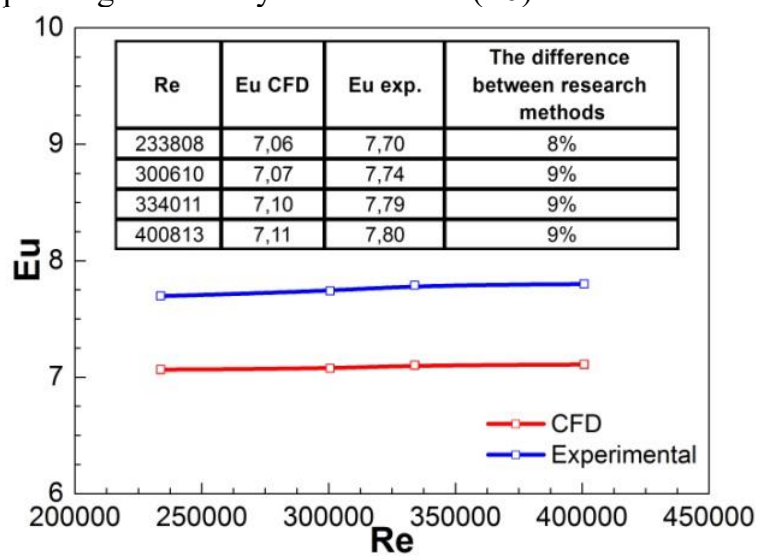

Fig. 3. Comparative analysis of $E u$ values depending on the research method (Mesh 2).

\section{Results and discussion}

\subsection{Vector of the flow field}

The two-phase flow in cyclone separators is strongly turbulent. The cross-section of traditional, circular cyclone structures contains two vortexes: the outer (free) vortex where 
fluid swirls down till the bottom and the inner (forced) vortex where the fluid is directed upward toward the vortex finder. This reflects the Rankine vortex, which is very beneficial for the separation of solid particles. The vector field shown in Fig. 4a indicates that a similar phenomenon also occurs in a square cyclone. An outer downward vortex is clearly visible near the cyclone wall, whereas in the middle section, the fluid moves upwards towards the vortex finder. The same phenomenon also occurs for vectors obtained through DPIV and CFD. In the case of DPIV, a slightly higher velocity was observed near the axis of the vortex finder (close to the inlet section). The velocity in this area decreased towards the walls of the vortex finder (which may have been caused by strong turbulences and the fact that the measurement system did not take into account all seeding particles). With both methods, the vectors had nearly identical directions.

The vector field shown in Fig. $4 \mathrm{~b}(Z / D=0.25)$ indicates that the maximal values of velocity occurred near the cyclone walls, whereas lower velocities occurred in the cyclone axis. This is also confirmed by the presence of the Rankine vortex. The fact that the central vortex was not located in the centre of the cyclone may be due to an unsteady oscillating flow near a precessing vortex core (PVC) in the central region. Furthermore, small local vortexes appeared in the corners of the walls due to a sharp turn in the flow. The geometry of the square cyclone in this region enforces a sharp, abnormal turn of the two-phase mixture, causing intense collisions between the particles and between the walls and the particles. Corners are one of the primary regions that cause pressure drop. Corners were found to benefit particle separation, mainly due to the strongly fluctuating flow absorbing a large portion of the kinetic energy of both the particles and the fluid [37, 38].

Figure 5 complements the vector analysis by showing velocity vectors in the centre of the inlet section of the cyclone separator. In this case, after the mixture stream left the inlet section, it encountered a significant obstacle, i.e. the external wall of the vortex finder, which caused the velocity to increase considerably in this region. The results obtained with CFD and DPIV were also very similar in this case. The distribution of velocity vectors in the inlet section obtained with DPIV indicates variations in velocity magnitude along the entire width.
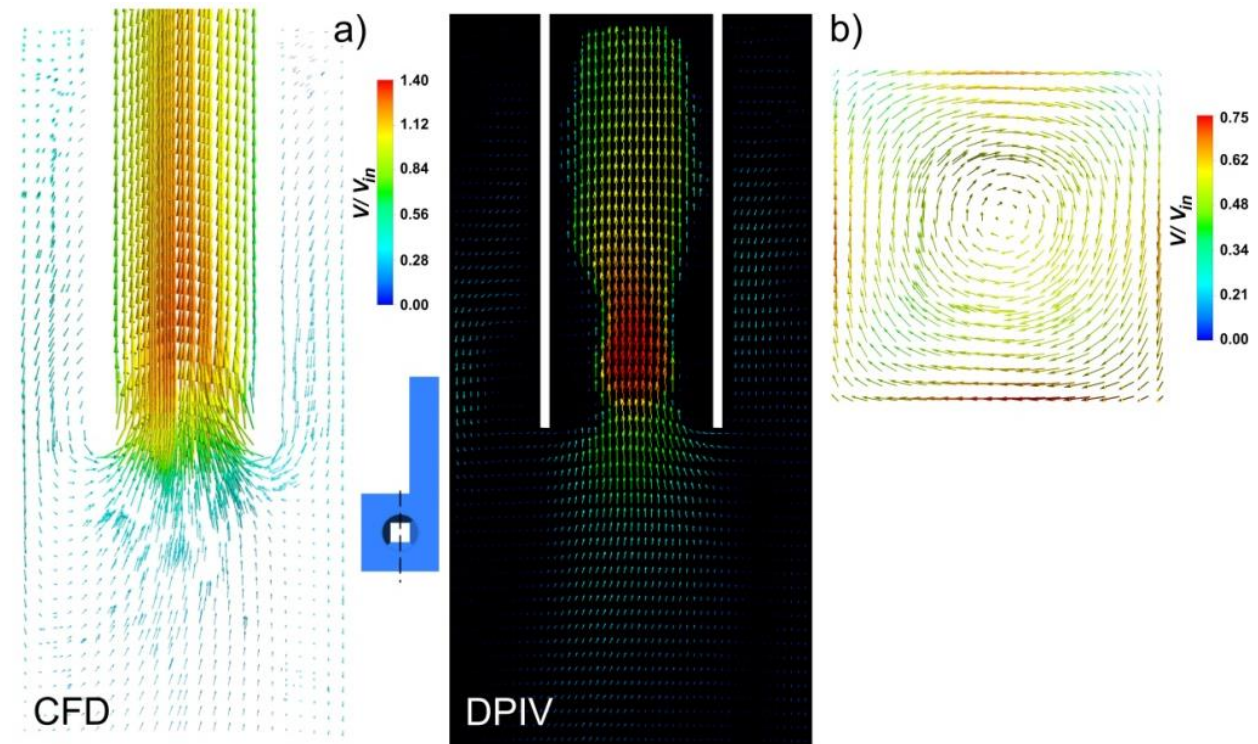

Fig. 4. Velocity vectors in middle of the inlet section (a) and at locations $Z / D=0.25$ (CFD) (b). 

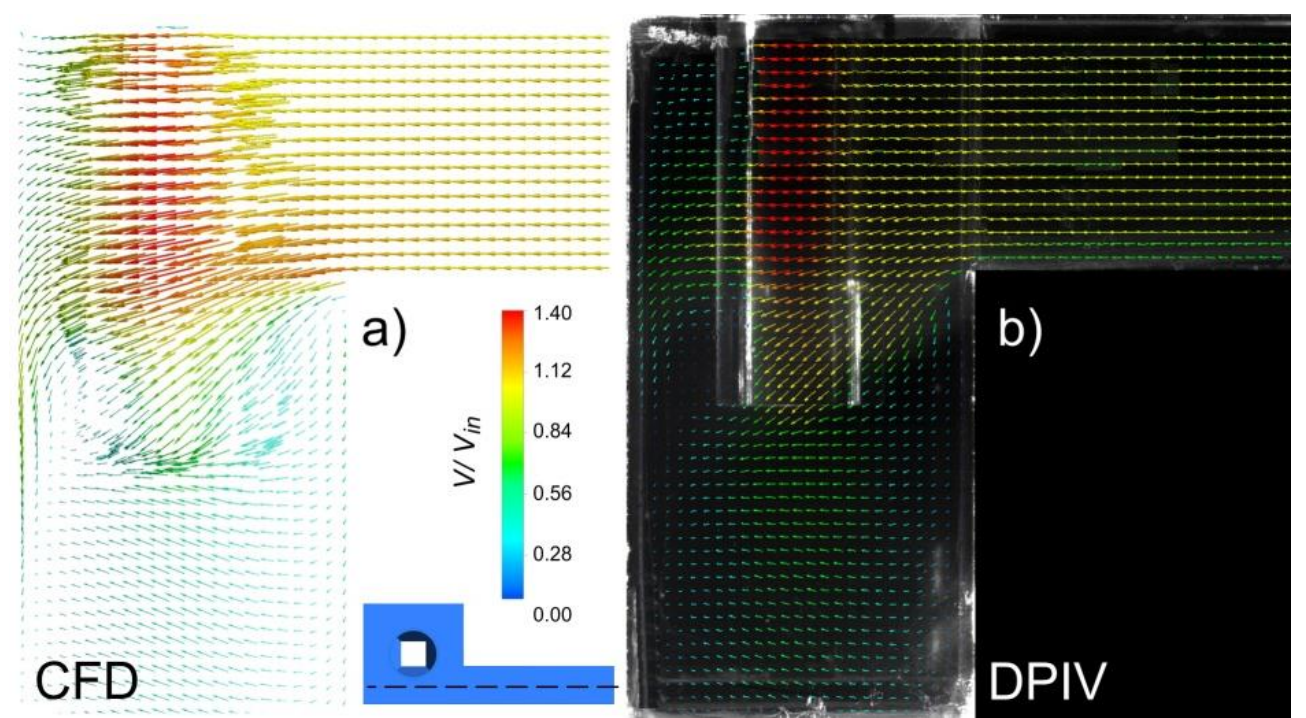

Fig. 5. Velocity vectors in center of the inlet section: (a) - CFD, (b) - DPIV.

\subsection{Mean axial and tangential velocity}

In traditional cyclone separators, axial velocity affects the movement of solid particles inside the cyclone: the fluid and the axial velocity of the external vortex cause them to move downwards and rotate near the walls. Figures 6 indicate that a similar phenomenon occurs in a square cyclone. Axial velocity is negative in the outer vortex region (a downward flow) and positive in the internal vortex region (an upward flow directed towards the vortex finder). The axial velocity contours presented here for both CFD and DPIV are very similar. However, note that DPIV yielded higher maximal and minimal values of the parameter.

A crucial constituent of the flow field in cyclone separators is tangential velocity, as it characterizes the efficiency of these devices by significantly affecting centrifugal force and, consequently, separation efficiency. The greater the centrifugal force in the radial direction, the more particles are directed towards the walls (and the fewer particles are directed towards the vortex finder). Figure $6 \mathrm{~b}$ (bottom row) shows the mean tangential velocity profile in two planes $(Z / D=0.5$ and $Z / D=0.25)$. In this case, the shape of the obtained curves also corresponded to cyclones with the traditional structure; this is consistent with the principles of rotational flow. The profiles in both sections are similar in shape (for both methods). They only differ in maximal and minimal values. Tangential velocity is much higher near the walls and approaches zero in the centre of the cyclone. The aim of the strongly eddying flow near the walls is to direct solid particles towards the walls, thus allowing for their separation. Positive values were observed on the left side, and negative values were observed on the right side. Tangential velocity in DPIV also reached higher maximal and minimal values. 

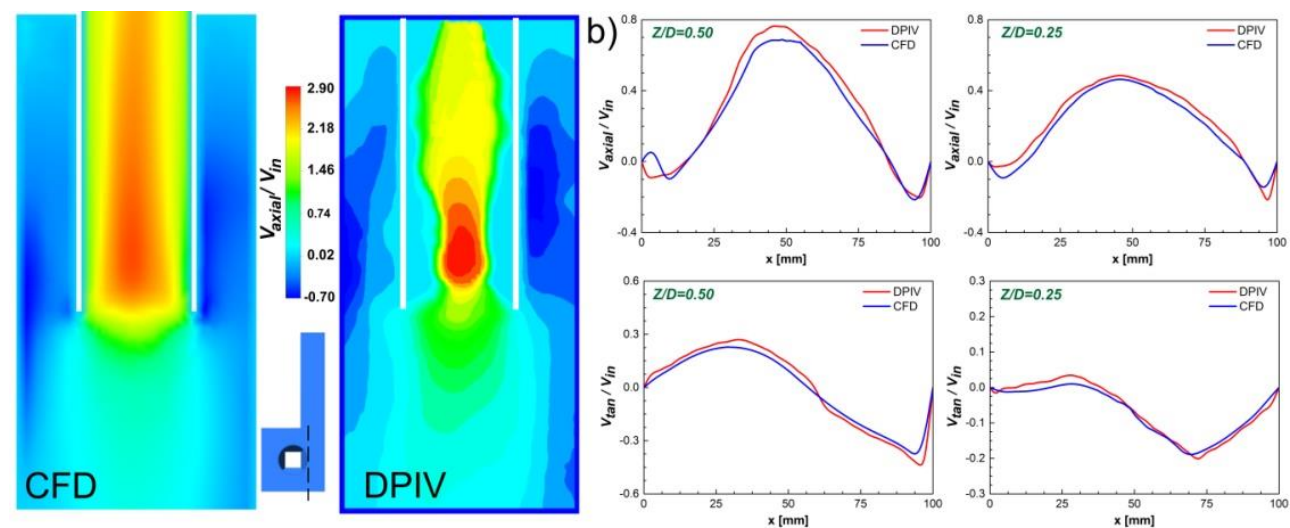

Fig. 6. Contour plots of mean axial velocity in the central section (a) and radial profiles (b) of mean axial velocity (top row) and mean tangential velocity (bottom row) at locations $Z / D=0.5$ and $Z / D=0.25$.

\section{Conclusion}

Two methods were employed to research the flow field inside a square cyclone separator, i.e. CFD and DPIV. The former is commonly used to analyze flow inside cyclone separators. However, in order to be reliable, the obtained results must be validated through a comparison to experimental measurements, which is hindered by the lack of a possibility to compare the obtained flow field distributions. This study has led to the following conclusions:

- The obtained vector fields and profiles of axial and tangential velocities for a square cyclone separator indicate that the flow inside a square cyclone has two constituents: an external, free flow and an internal, forced flow in the centre, as with cyclones with the traditional structure;

- The corners of a square cyclone general additional vortexes that cause pressure drop to increase (but may, at the same time, potentially improve separation efficiency);

- Slightly higher maximal and minimal values of the analyzed parameters were obtained for DPIV;

- The obtained results are highly consistent between both methods (and, consequently, constitute good methods for researching flow in square cyclones and can be used to optimize it);

- DPIV may also be a very good method for validating results obtained with CFD, including for the analysis of flow fields and other parameters of flow.

As a future work, we plan to extend the work by employing both DPIV and CFD (preferably LES) to enhance the understanding of the flow physics and to optimize the geometry of square cyclone.

This research was financially supported by the scientific activity number 2018/02/X/ST8/01645 from the National Science Centre, Poland (Narodowe Centrum Nauki - NCN).

\section{References}

1. L. K. Wang, N. C. Pereira, Y. T. Hung, Air Pollution Control Engineering (Totowa, 2004) 
2. J. Dirgo. D. Leith. Aerosol Sci. Tech. 4 (1985)

3. B. Wang, D. L. Xu, K. W. Chu, A. B. Yu, App. Math. Model. 30 (2006)

4. K. Elsayed, C. Lacor, Appl. Math. Model. 35 (2011).

5. L. S. Brar, R. P. Sharma, K. Elsayed, Powder Technol. 286 (2015)

6. C. W. Haig, A. Hursthouse, D. Sykes, S. Mcilwain, Appl. Math. Model. 40 (2016)

7. L. S. Brar, K. Elsayed, Sep. Purif. Technol. 207 (2018)

8. L. Brar, Today: Proceedings 5 (2018)

9. V. Kumar, K. Jha, J. Mech. Sci. Technol. 11 (2018)

10. R. Gamble, T. Hyppanen, T. Kauranen, Proceeding of the $12^{\text {th }}$ International Conference on Fluidized Bed Combustion (1993)

11. G. X. Yue, Y. Li, X. X. Zhao, Proceeding of the $14^{\text {th }}$ International Conference on Fluidized Bed Combustion (1997)

12. S. Wang, M. Fang, Z. Luo, X. Li, M. Ni K, Cen. Powder Technol. 102 (1999)

13. J. F. Lu, G. X. Yu, Q. Liu, Proceeding of the $15^{\text {th }}$ International Conference on Fluidized Bed Combustion, 1999

14. H. Fatahian, E. Fatahian, M. E. Nimvari, Powder Technol. 339 (2018)

15. B. Dobrowolski, M. Skulska, J.Wydrych, Archiwum Energetyki 38 (2008)

16. M. Thulasiraman, K. Pitchandi, J. Mech. Sci. Technol. 29 (2015)

17. F. Mariani, F. Risi, C. Grimaldi, Sep. Purif. Technol. 179 (2017)

18. E. Kashani, A. Mohebbi, M. G. Heidari, Powder Technol. 327 (2018)

19. M. Wasilewski, L. S. Brar, Sep. Purif. Technol. 213 (2019)

20. D. Misiulia, K. Elsayed, A. G. Andersson, Sep. Purif. Technol. 185 (2017)

21. M. Wasilewski, G. Ligus, E3S Web of Conferences 0018544 (2018)

22. S. Pirker, C. Goniva, C. Kloss, S. Puttinger, J. Houben, S. Schneiderbauer, Powder Technol. 235 (2013)

23. L. S. Brar, K. Elsayed, Powder Technol. 311 (2017)

24. R. J. Adrian, J. Westerweel, Cambridge University Press (2011)

25. C. E. Willert, Apply. Sci. Res. 56 (1996)

26. K. H. Leea, C. H. Leea, C. S. Leeb, Fuel 83 (2004)

27. C. Iwaki, K. H. Cheong, H. Monji, G. Matsui, Exp. Fluids 37 (2004)

28. J. Westerweel. Exp. Fluids 29 (2000)

29. S. S. Paul, M. F. Tachie, S. J. Ormiston, Int. J. Heat Fluid Fl. 28 (2007)

30. Ch. Bin, G. Liejin, J. Therm. Sci. 9 (2012)

31. G. Ligus, Sz. Kołodziej, E3S Web of Conferences 0100519 (2017)

32. Sz. Kołodziej, G. Ligus, E3S Web of Conferences 0101719 (2017)

33. G. Ligus, M. Wasilewski, E3S Web of Conferences 0009644 (2018)

34. Z. Liu, Y. Zheng, L. Jia, J. Jiao, Q. Zhang, Chem. Eng. Sci. 61 (2006)

35. Z. Liu, J. Jiao, Y. Zheng, Q. Zhang, AIChE J. 52 (2006)

36. B. Zhang, S. Hui, International Conference on Power Engineering (2007)

37. Y. Su, Y. Mao, Chem. Eng. J. 121 (2006)

38. K. Qiu, J. Yan, X. Li, Journal of Engineering for Thermal Energy and Power 14 (1999) 